\title{
Introdução precoce da alimentação complementar infantil: comparando mães adolescentes e adultas
}

Early introduction of complementary feeding in infants: comparing adolescent and adult mothers Introducción precoz de alimentación complementaria infantil: comparación entre madres adolescentes y adultas

Carla Porto Cunha Murari ${ }^{1}$ iD https://orcid.org/0000-0002-8322-6165

Ana Paula Rodrigues Arciprete ${ }^{1}$ is https://orcid.org/0000-0002-0353-4820

Flávia Gomes-Sponholz ${ }^{1}$ io https://orcid.org/0000-0003-1540-0659 Juliana Cristina dos Santos Monteiro ${ }^{1}$ io https://orcid.org/0000-0001-6470-673X F, Monteiro JC. Introdução precoce da alimentação complementar infantil: comparando mães adolescentes e adultas. Acta Paul Enferm. 2021;34:eAPE01011.

DOI http://dx.doi.org/10.37689/actaape/2021A001011

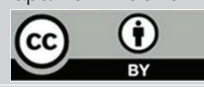

Aleitamento materno; Desmame precoce; Nutrição da criança; Saúde Materno-infantil; Adolescência

Keywords

Beast feeding; Weaning: Child nutrition; Materna and child health; Adolescent

Descriptores

Lactancia mater; Destete; Nutrición del niño; Salud materno-infantil; Adolescente

\section{Submetido \\ 28 de Junho de 2019 \\ Aceito \\ 1 de Junho de 2020}

Autor correspondente

Ana Paula Rodrigues Arciprete

E-mail: paula_arciprete@yahoo.com.br

\section{Resumo}

Objetivo: Verificar a associação entre a idade materna e os motivos alegados pelas mães para o início precoce da alimentação complementar aos 30, 90 e 180 dias de vida da criança.

Métodos: Trata-se de um estudo de coorte prospectivo, desenvolvido em uma maternidade do Sistema Único de Saúde de um município de médio porte do interior do Estado de São Paulo, Brasil. A amostra foi de 545 participantes, sendo 103 adolescentes e 442 adultas no período pós-parto. Os dados foram coletados de outubro de 2016 a outubro de 2017, face-a-face na maternidade e via telefone em 30, 90 e 180 dias após 0 pato, e analisados por meio dos testes Qui-Quadrado e Exato de Fisher.

Resultados: Aos 30 dias pós-parto as adolescentes referiram motivos para introdução de chá relacionados às orientações de outras pessoas; já as adultas, referiram motivos relacionados à criança $(p=0,001)$. Aos 90 dias, para a introdução de água e chá, as adolescentes alegaram orientações de outras pessoas e as adultas alegaram motivos da criança ( $p=0,004$ para água e $p<0,001$ para chá). Aos 180 dias, para a introdução de outro leite, adolescentes e adultas referiram motivos relacionados ao seu próprio desejo $(p=0,03)$.

Conclusão: As adolescentes e adultas apresentaram motivos diferentes para a introdução de chá aos 30 dias pós-parto, para a introdução de água e chá aos 90 dias e apresentaram o mesmo motivo para a introdução de outro leite aos 180 dias pós-parto.

\section{Abstract}

Objective: Check for associations between maternal age and the reasons provided by mothers for the early introduction of complementary feeding in infants at 30,90 and 180 days after birth.

Methods: This is a prospective cohort study, conducted in a maternity hospital of the Brazilian Unified Health System (SUS) located in a medium-sized city of the State of São Paulo, Brazil. The sample was made up of 545 participants - 103 adolescents and 442 adults in the postpartum period. Data were collected from October 2016 to October 2017, face to face, at the maternity hospital and via telephone 30, 90 and 180 days after birth, and analyzed using the Chi-square and Fisher's exact tests.

Results: Thirty days after birth, the adolescent mothers mentioned recommendations of other people for introducing tea into the baby's diet; on the other hand, the adult mothers mentioned reasons related to the child $(p=0.001$ ). Ninety days after birth, the adolescent mothers reported recommendations for the introduction of water and tea from other people and the adult mothers reported reasons related to the child $(p=0.004$ for water and $p<0.001$ for tea). And 180 days after birth, the adolescent and adult mothers reported reasons related to their own choice for the introduction of another type of milk ( $p=0.03$ ). 
Conclusion: Adolescent and adult mothers presented different reasons for introducing tea 30 days after birth, for introducing water and tea 90 after birth, and presented the same reason for introducing another type of milk 180 days after birth.

\section{Resumen}

Objetivo: Verificar la relación entre la edad materna y los motivos indicados por las madres para el inicio precoz de la alimentación complementaria a los 30, 90 y 180 días de vida del bebé.

Métodos: Se trata de un estudio de cohorte prospectivo, llevado a cabo en una maternidad del Sistema Único de Salud de un municipio de tamaño medio en el interior del estado de São Paulo, Brasil. La muestra fue de 545 participantes, de las cuales 103 eran adolescentes y 442 adultas en el período de posparto. Los datos fueron recopilados de octubre de 2016 a octubre de 2017, cara a cara en la maternidad y por teléfono a los 30,90 y 180 días después del parto, y fueron analizados mediante la prueba $\chi^{2}$ de Pearson y la prueba exacta de Fisher.

Resultados: A los 30 días de posparto, las adolescentes mencionaron motivos para la introducción de té relacionados con instrucciones de otras personas. Las adultas mencionaron motivos relacionados con el bebé $(p=0,001)$. A los 90 días, para la introducción de agua y té, las adolescentes declararon que siguieron instrucciones de otras personas y las adultas que fue por motivos del bebé ( $p=0,004$ para agua y $p<0,001$ para té). A los 180 días, para la introducción de otra leche, las adolescentes y las adultas mencionaron motivos relacionados con su propio deseo $(p=0,03)$.

Conclusión: Las adolescentes y adultas presentaron motivos diferentes para la introducción de té a los 30 días posparto y para la introducción de agua y té a los 90 días, y presentaron el mismo motivo para la introducción de otra leche a los 180 días posparto.

\section{Introdução}

$\mathrm{O}$ aleitamento materno (AM) mantém-se como preocupação para a saúde pública, visto que em diferentes países seus índices não atingem as recomendaçôes da Organização Mundial da Saúde (OMS), mesmo com todo o conhecimento sobre os benefícios já evidenciados na literatura científica. ${ }^{(1)}$ A introdução precoce de alimentos complementares na dieta de uma criança diminui a duração do $\mathrm{AM}$, interfere na absorção de nutrientes considerados importantes, como o ferro, reduz a eficácia da lactação no intervalo intergestacional e aumenta a morbimortalidade infantil. (2)

No Brasil, é grande o número de crianças que consomem vários tipos de alimentos antes dos seis meses, diferentemente do que é recomendado pela OMS. ${ }^{(2)}$ De acordo com alguns estudos, a falta de informação das mulheres sobre a composição e qualidade do leite humano impede que elas reconheçam os benefícios do aleitamento materno, fazendo com que iniciem a alimentação complementar de seus filhos precocemente. ${ }^{(3-5)}$

A introdução de outros leites de forma precoce na alimentação dos filhos é justificada pelas mães, principalmente, por motivos relacionados à percepção de quantidade insuficiente e baixa qualidade do leite materno. ${ }^{(6)}$ No entanto, sabe-se que na população mundial a maioria das mulheres apresenta condiçôes biológicas de produzir leite em quantidade suficiente para atender as necessidades de seu filho, sendo que o índice de hipogalactia não ultrapassa
$1,5 \%$ e a produção de leite de baixo valor nutricional é situação rara. ${ }^{(7)}$

Considerando-se a cultura brasileira, existem crenças e práticas impregnadas no comportamento da população que são conflitantes com as recomendaçóes para alimentação do lactente como, por exemplo, a oferta de água para saciar a sede e chás para acalmar, aliviar cólicas ou tratar diferentes doenças. Tais crenças, aliadas àquela de que o leite materno não é completo para suprir as necessidades da criança, também podem resultar na introdução de substitutos do leite materno precocemente na dieta das crianças. ${ }^{(8)}$

Outro fator que influencia a introdução precoce da alimentação complementar é a idade materna, em especial, mães jovens. ${ }^{(9)}$ Autores demonstram que mães adolescentes amamentam seus filhos por menos tempo quando comparadas às máes adultas, devido a fatores como a existência ou náo de vida conjugal e o retorno à vida escolar. ${ }^{(4)}$ Alguns estudos evidenciaram que mães adolescentes apresentam mais chance para desmamar seus filhos precocemente. ${ }^{(5-10)}$ Entretanto, os motivos que levam as adolescentes a desmamarem não estão evidentes na literatura científica, o que chama a atenção para a necessidade de entender quais são as particularidades da maternidade e da amamentação na fase adulta e na adolescência. $\mathrm{O}$ entendimento dos motivos revelados por mães adolescentes e adultas pode contribuir para a implementação de ações específicas mais efetivas no incentivo e no apoio à amamentação desses dois grupos de mulheres. 
Este estudo tem como objetivo verificar a associação entre a idade materna e os motivos alegados pelas mães para o início precoce da alimentação complementar aos 30, 90 e 180 dias de vida da criança.

\section{Métodos}

Trata-se de um estudo de coorte prospectivo, desenvolvido em uma maternidade do Sistema Único de Saúde de um município de médio porte do interior do Estado de São Paulo, Brasil.

A população de referência foi constituída por todas as mulheres adolescentes e adultas no período pós-parto, admitidas na enfermaria da referida maternidade. $\mathrm{O}$ cálculo amostral foi realizado com informaçôes do Relatório Anual de Enfermagem da instituição, baseado no acompanhamento longitudinal mensal das unidades amostrais selecionadas para os grupos de adolescentes e adultas. Considerou-se erro amostral tolerável de 5\%, nível de confiança de $95 \%$, perda prevista de $10 \%$ e os grupos de adolescentes e adultas. A amostra final calculada foi de 545 participantes, sendo 103 adolescentes e 442 adultas.

As participantes foram selecionadas de acordo com os critérios de inclusão: puérperas adolescentes e adultas com bebês que estavam em condiçóes biológicas para amamentar, que tiveram filhos com idade gestacional a termo, que não necessitavam amamentar com ajuda de sondas ou intermediários, que estavam acompanhadas pelos seus filhos no alojamento conjunto e que tinham no mínimo vinte $\mathrm{e}$ quatro horas de pós-parto para início da pesquisa. Os critérios de exclusão foram: puérperas ou bebês que apresentavam patologias ou intercorrências que impediam o aleitamento materno e puérperas com deficiência auditiva, visual ou cognitiva.

Para coleta de dados foram utilizados dois instrumentos. $\mathrm{O}$ primeiro instrumento foi construído especificamente para este estudo e contempla, de forma estruturada, os dados de identificação e as características sociodemográficas, obstétricas e de amamentação. Este instrumento foi utilizado para a entrevista face a face com a mulher na materni- dade. O segundo instrumento de coleta de dados foi composto por questóes fechadas para identificar a alimentação oferecida ao bebê e uma questão aberta, para a identificação dos motivos da introdução de alimentos complementares. Esse segundo instrumento foi utilizado por meio de contato via telefone, em 30, 90 e 180 dias após o parto. O contato telefônico nestes períodos justifica-se, pois no primeiro mês pós-parto as nutrizes podem vivenciar dificuldades e obstáculos estando suscetíveis ao desmame precoce; no terceiro mês pode-se avaliar se houve a superação destas dificuldades e no sexto mês é possível avaliar se houve a manutenção do AME conforme recomendação da OMS. O contato telefônico foi realizado de acordo com as informaçóes fornecidas pelas participantes durante a aplicação do primeiro instrumento de coleta de dados. Nos casos em que a participante não foi encontrada, foram realizadas até três tentativas em dias e horários diferentes antes de serem excluídas da pesquisa. Assim, 18 participantes deixaram de ser acompanhadas.

A coleta de dados ocorreu no período de outubro de 2016 a outubro de 2017. Seguindo os critérios estabelecidos, as puérperas foram convidadas a participar do estudo, sendo esclarecidas sobre o mesmo. Após terem ciência da pesquisa e dos aspectos éticos, as puérperas adultas que aceitaram participar assinaram o Termo de Consentimento Livre e Esclarecido (TCLE). As puérperas adolescentes que aceitaram participar assinaram o Termo de Assentimento, sendo que foi solicitada também a autorização de seus responsáveis legais, que assinaram um TCLE autorizando a participação das adolescentes.

Nesta pesquisa, foram utilizadas as seguintes definiçóes:

- Adolescência: período que compreende dos 10 aos 19 anos. ${ }^{(9)}$

- Aleitamento materno exclusivo (AME): quando a criança recebe somente leite materno, direto da mama ou ordenhado, ou leite humano de outra fonte, sem outros líquidos ou sólidos com exceção de gotas ou xaropes contendo vitaminas, sais minerais de reidratação oral, suplementos minerais ou medicamentos. ${ }^{(11)}$ 
- Aleitamento materno: quando a criança recebe leite materno direto da mama ou ordenhado, independentemente de receber ou não outros alimentos. $^{(11)}$

- Alimentação complementar oportuna: introdução de alimentos sólidos, semissólidos ou líquidos oferecidos à criança em adição ao leite materno entre os seis e sete meses. ${ }^{(12)}$

- Desmame precoce: quando a criança deixa de receber o leite materno antes dos seis meses de vida. $^{(4)}$

Os dados foram armazenados em uma planilha eletrônica estruturada no Microsoft Excel, por meio de dupla digitação, o que possibilitou a validação dos dados digitados para eliminar possíveis erros e garantir confiabilidade na compilação. Foram analisados com a utilização do programa estatístico $\mathrm{R}$ - versão 3.4.2. ${ }^{(13)}$ Para todas as análises estatísticas, foram considerados significativos valores de $p$ menores que 0,05 .

O estudo foi autorizado pela maternidade e aprovado por Comitê de Ética em Pesquisa vinculado a Comissão Nacional de Ética em Pesquisa (CONEP) do Conselho Nacional de Saúde com protocolo CAAE: no 56833316.0.0000.5393.

\section{Resultados}

As participantes totalizaram 103 mães adolescentes e 442 adultas. A caracterização sociodemográfica das participantes está apresentada na tabela 1 .

Entre as adolescentes, a média foi de 1,31 gestação, 1,2 parto, 1,11 aborto, 1,19 filho vivo, média de 16,81 semanas de idade gestacional no início do pré-natal e média de 6,73 consultas de pré-natal. Entre as puérperas adultas, a média foi de 2,44 gestaçóes, 2,26 partos, 1,13 aborto, 2,23 filhos vivos, média de 14,5 semanas de idade gestacional no início do pré-natal e média de 7,73 consultas de pré-natal. A maioria das participantes, tanto adolescentes quanto adultas, não tinha planejado a gestação $(70,3 \%)$. Dentre as mulheres investigadas, a maioria delas não apresentou intercorrências durante a gestação $(56,0 \%)$, parto $(90,4 \%)$ e pós-parto
Tabela 1. Distribuição das participantes do estudo segundo idade, cor autorreferida, escolaridade, interferência da gestação nos estudos, religião, ocupação, estado marital, moradia, auxílio nos cuidados com o bebê e renda familiar em salários mínimos de $\mathrm{R} \$ 890,00$

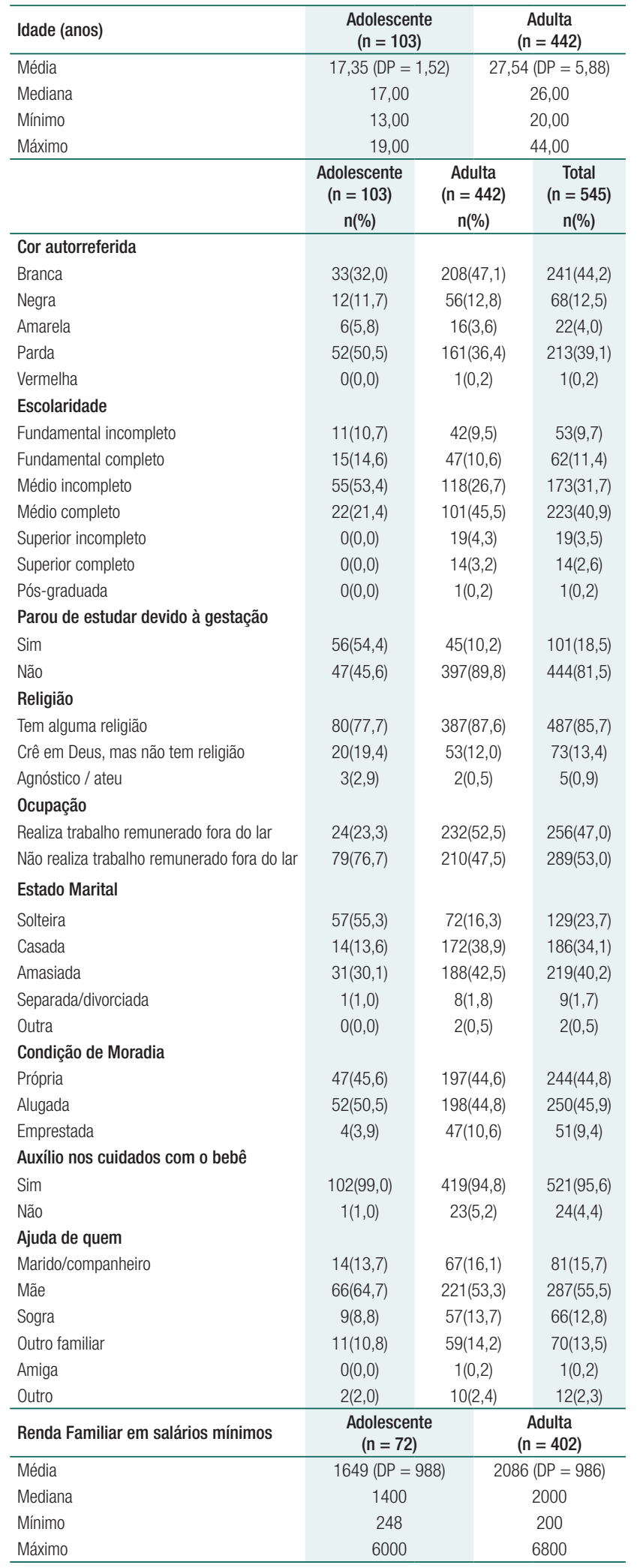


$(64,8 \%)$. Com relação ao tipo de parto, $51,5 \%$ das adolescentes teve parto normal e 56,5\% das adultas foram submetidas à cesariana. No que concerne às características dos recém-nascidos (RN) filhos das participantes, 52,8\% eram do sexo feminino, $88,1 \%$ foram amamentados na primeira hora de vida e 90,6\% estavam em AME no momento da coleta de dados na maternidade. A média de peso dos $\mathrm{RN}$ ao nascimento entre as adolescentes foi de 3,089 kg e, entre as adultas, 3,197 kg. A prevalência do AME no período investigado pode ser observada na tabela 2 .

Tabela 2. Prevalência do aleitamento materno exclusivo em 30 , 90 e 180 dias pós-parto

\begin{tabular}{|c|c|c|c|c|}
\hline & $\begin{array}{l}\text { Adolescente } \\
(n=103)\end{array}$ & $\begin{array}{c}\text { Adulta } \\
(\mathrm{n}=442)\end{array}$ & $\begin{array}{c}\text { Total } \\
(n=545)\end{array}$ & $p$-valor* \\
\hline & $\mathrm{n}(\%)$ & $n(\%)$ & $n(\%)$ & \\
\hline \multicolumn{5}{|l|}{$\begin{array}{l}\text { AME } \\
\text { (30 dias pós-parto) }\end{array}$} \\
\hline $\begin{array}{l}\text { Sim } \\
\text { Não } \\
\text { Desmame precoce }\end{array}$ & $\begin{array}{c}50(48,5) \\
46(44,7) \\
7(6,8)\end{array}$ & $\begin{array}{c}270(61,1) \\
125(28,3) \\
47(10,6)\end{array}$ & $\begin{array}{c}320(58,7) \\
171(31,4) \\
54(9,9)\end{array}$ & 0,004 \\
\hline \multicolumn{5}{|l|}{$\begin{array}{l}\text { AME } \\
\text { (90 dias pós-parto) }\end{array}$} \\
\hline $\begin{array}{l}\text { Sim } \\
\text { Não } \\
\text { Desmame precoce }\end{array}$ & $\begin{array}{l}22(21,4) \\
64(62,1) \\
17(16,5)\end{array}$ & $\begin{array}{c}180(40,7) \\
183(41,4) \\
79(17,9)\end{array}$ & $\begin{array}{c}202(37,1) \\
247(45,3) \\
96(17,6)\end{array}$ & 0,000 \\
\hline \multicolumn{5}{|l|}{$\begin{array}{l}\text { AME } \\
\text { (180 dias pós - parto) }\end{array}$} \\
\hline $\begin{array}{l}\text { Sim } \\
\text { Não } \\
\text { Desmame precoce }\end{array}$ & $\begin{array}{l}11(10,7) \\
59(57,3) \\
33(32,0)\end{array}$ & $\begin{array}{c}71(16,1) \\
233(52,7) \\
138(31,2)\end{array}$ & $\begin{array}{c}82(15,0) \\
292(53,6) \\
171(31,4)\end{array}$ & 0,200 \\
\hline
\end{tabular}

Quanto à alimentação oferecida ao filho em 30, 90 e 180 dias pós-parto, identificamos os seguintes alimentos oferecidos: leite do peito, outro tipo de leite, água, chá e fruta, na forma de suco e/ ou papa. Na tabela 3 estão apresentados os motivos alegados para o início da alimentação complementar precoce com 30 dias pós-parto entre mães adolescentes a adultas e seus respectivos valores de significância estatística. O chá foi o alimento mais oferecido; quanto à decisão para a introdução precoce de chá na dieta da criança, as adolescentes alegaram seguir sugestôes ou indicaçôes de terceiros, como prescrição médica ou orientação de alguém da família (55,2\%). Já entre as adultas os principais motivos estavam relacionados à sua percepção de que havia a necessidade por uma demanda da criança, a partir da observação de seu comportamento (80,9\%).
Tabela 3. Motivos alegados para a introdução de outros alimentos na dieta da criança com 30 dias, entre mães adolescentes e adultas

\begin{tabular}{|c|c|c|c|}
\hline Motivos & $\begin{array}{c}\text { Adolescente } \\
\mathrm{n}(\%)\end{array}$ & $\begin{array}{c}\text { Adulta } \\
\mathrm{n}(\%)\end{array}$ & $p$-value ${ }^{*}$ \\
\hline \multicolumn{4}{|l|}{ Introdução de água } \\
\hline Relacionados à criança & $13(41,9)$ & $25(58,1)$ & 0,080 \\
\hline Relacionados à mulher/mãe & $0(0,0)$ & $3(7,0)$ & \\
\hline Outros (médico/família) & $18(58,1)$ & $15(34,9)$ & \\
\hline \multicolumn{4}{|l|}{ Introdução de chá } \\
\hline Relacionados à criança & $13(44,8)$ & $55(80,9)$ & $0,001^{\star \star}$ \\
\hline Relacionados à mulher/mãe & - & - & - \\
\hline Outros (médico/família) & $16(55,2)$ & $13(19,1)$ & \\
\hline \multicolumn{4}{|l|}{ Introdução de leites artificiais } \\
\hline Relacionados à criança & $1(4,0)$ & $8(7,4)$ & 1,0 \\
\hline Relacionados à mulher/mãe & $18(72,0)$ & $76(70,4)$ & \\
\hline Outros (médico/família) & $6(24,0)$ & $24(22,2)$ & \\
\hline
\end{tabular}

* Teste Exato de Fisher; *^ Teste Qui-quadrado

Como observado, aos 30 dias, os motivos alegados pelas adolescentes para a introdução de chá estavam associados à orientação de outras pessoas; para as adultas, os motivos estavam associados à percepção das mães sobre a necessidade do filho $(p=0,001)$. Na tabela 4 estáo apresentados os motivos alegados pelas participantes para iniciar a alimentação complementar precoce com 90 dias pós-parto entre mães adolescentes e adultas. A água foi o complemento mais oferecido; quanto à decisão para a introduçáo precoce de água na dieta do filho, as adolescentes alegaram ter seguido sugestóes ou indicações de terceiros, como prescrição médica ou orientação de alguém da família $(51,6 \%)$ e, entre as adultas, os principais motivos estavam relacionados à sua percepção de que havia a necessidade por uma demanda da criança $(68,0 \%)$.

Aos 90 dias os motivos alegados pelas adolescentes para a introdução de água estiveram relacionados à orientação de outras pessoas; entre as adultas, os motivos estavam relacionados à criança $(p=0,004)$. Em relação à introdução de chá, as adolescentes alegaram como motivo a orientação médica e/ou de familiares e as adultas alegaram motivos relacionados à criança $(\mathrm{p}<0,001)$. Na tabela 5 estão apresentados os motivos alegados pelas participantes para introdução precoce de alimentos na dieta das crianças com 180 dias pós-parto. A fruta foi o alimento mais oferecido, em forma de suco e/ou papa. Adolescentes e adultas alegaram ofertar frutas por sugestão e ou orientação de outras pessoas. 
Tabela 4. Motivos alegados para a introdução de outros alimentos na dieta da criança com 90 dias, entre mães adolescentes e adultas

\begin{tabular}{|c|c|c|c|}
\hline Motivos & $\begin{array}{c}\text { Adolescente } \\
\mathrm{n}(\%)\end{array}$ & $\begin{array}{l}\text { Adulta } \\
\mathrm{n}(\%)\end{array}$ & p-valor* \\
\hline \multicolumn{4}{|l|}{ Introdução da água } \\
\hline Relacionados à criança & $29(45,3)$ & $100(68,0)$ & 0,004 \\
\hline Relacionados à mulher/mãe & $2(3,1)$ & $5(3,4)$ & \\
\hline Outros (médico/família) & $33(51,6)$ & $42(28,6)$ & \\
\hline \multicolumn{4}{|l|}{ Introdução da chá } \\
\hline Relacionados à criança & $7(36,8)$ & $44(83,0)$ & $0,000^{\star *}$ \\
\hline Relacionados à mulher/mãe & - & - & \\
\hline Outros (médico/família) & $12(63,2)$ & $9(17,0)$ & \\
\hline \multicolumn{4}{|l|}{ Introdução da suco } \\
\hline \multicolumn{4}{|l|}{ Relacionados à criança } \\
\hline Relacionados à mulher/mãe & $0(0,0)$ & $1(33,3)$ & 1,000 \\
\hline Outros (médico/família) & $3(100,0)$ & $2(66,7)$ & \\
\hline \multicolumn{4}{|l|}{ Introdução da leite } \\
\hline Relacionados à criança & $1(2,9)$ & $6(4,1)$ & 0,184 \\
\hline Relacionados à mulher/mãe & $25(73,5)$ & $122(84,1)$ & \\
\hline Outros (médico/família) & $8(23,5)$ & $17(11,7)$ & \\
\hline \multicolumn{4}{|l|}{ Introdução da papa ou fruta } \\
\hline \multicolumn{4}{|l|}{ Relacionados à criança } \\
\hline Relacionados à mulher/mãe & $0(0,0)$ & $2(40,0)$ & 1,000 \\
\hline Outros (médico/família) & $2(100)$ & $3(60,0)$ & \\
\hline
\end{tabular}

*Teste Exato de Fisher $\quad$ ** Teste Qui-quadrado

Tabela 5. Motivos alegados para o início da alimentação complementar precoce com 180 dias pós-parto, entre mães adolescentes e adultas

\begin{tabular}{lccc}
\hline Motivos & $\begin{array}{c}\text { Adolescente } \\
\mathbf{n}(\%)\end{array}$ & $\begin{array}{c}\text { Adulta } \\
\mathbf{n}(\%)\end{array}$ & p-valor* \\
\hline Água & & & \\
$\quad$ Relacionados à criança & $28(45,2)$ & $98(50,5)$ & 0,266 \\
$\quad$ Relacionados à mulher/mãe & $4(6,5)$ & $23(11,9)$ & \\
$\quad$ Outros (médico/família) & $30(48,4)$ & $73(37,6)$ & \\
Chá & & & \\
$\quad$ Relacionados à criança & - & $1(100)$ & 1,000 \\
$\quad$ Relacionados à mulher/mãe & - & - & \\
$\quad$ Outros (médico/família) & $1(100)$ & - & \\
Suco de fruta & & & \\
$\quad$ Relacionados à criança & - & $2(1,9)$ & 1,000 \\
$\quad$ Relacionados à mulher/mãe & $7(30,4)$ & $30(28,6)$ & \\
$\quad$ Outros (médico/família) & $16(69,6)$ & $73(69,5)$ & \\
Outro tipo de leite & & & \\
$\quad$ Relacionados à criança & $1(2,9)$ & $4(2,5)$ & 0,030 \\
$\quad$ Relacionados à mulher/mãe & $22(62,9)$ & $132(82,0)$ & \\
$\quad$ Outros (médico/família) & $12(34,3)$ & $25(15,5)$ & \\
Papa de fruta & & & \\
$\quad$ Relacionados à criança & $1(2,1)$ & $7(3,3)$ & 0,890 \\
Relacionados à mulher/mãe & $16(33,3)$ & $67(32,1)$ & \\
$\quad$ Outros (médico/família) & $31(64,6)$ & $135(64,6)$ & \\
\hline
\end{tabular}

${ }^{*}$ Teste Exato de Fisher

Aos 180 dias, as mães adolescentes e adultas referiram o mesmo motivo para a introdução de outro leite, que foi relacionado à própria mulher $(\mathrm{p}=0,03)$.

\section{Discussão}

Apesar dos vários efeitos adversos na saúde das crianças, a introdução precoce da alimentação complementar continua sendo comum tanto em países desenvolvidos quanto nos em desenvolvimento, assim como ocorreu na presente pesquisa. Nesse sentido, estudo multicêntrico europeu que incluiu cinco países (Bélgica, Alemanha, Itália, Polônia e Espanha) revelou que houve início de alimentação complementar antes do quarto mês em aproximadamente $25 \%$ das crianças investigadas; aos seis meses, pelo menos $90 \%$ das crianças já tinham consumido alimentos sólidos. ${ }^{(14)}$ Pesquisa sobre alimentação complementar no contexto do Oriente Médio revelou que as práticas contemporâneas não seguem as recomendações estabelecidas pela OMS; no Iraque, nos Emirados Árabes e no Líbano, 78,6\%, 70,0\% e 52,9\% das crianças, respectivamente, receberam alimentos complementares enquanto ainda estavam entre quatro e seis meses de idade. ${ }^{(15)}$

No presente estudo, com relação ao oferecimento de água nos primeiros 30 dias e ao oferecimento de água e chá aos 90 dias, verificou-se que a maioria das adolescentes alegaram motivos de prescrição médica e/ou orientação da família; já a maioria das adultas alegaram motivos relacionados à criança. No Brasil, chá e água são os líquidos mais frequentemente introduzidos precocemente na dieta da criança. Estudo realizado em Goiânia, com a maioria das participantes acima de 20 anos, apontou que logo no primeiro mês de vida da criança, o consumo de chá chegou a 32,6\% e de água a 19,1\%. (16) Este mesmo estudo mostrou que, além do consumo de água e chá desde o primeiro mês de vida, foi acentuado o consumo de água $(54,1 \%)$ e chás $(31,5 \%)$, acompanhado de outros leites $(18,0 \%)$ e sucos $(11,5 \%) .{ }^{(16)}$ Já o estudo realizado no interior do estado de São Paulo com crianças de aproximadamente quatro meses, demonstrou que a água foi o líquido mais oferecido pelas mães para saciar a sede dos seus filhos, ${ }^{(17)}$ ainda que, de acordo com a OMS, crianças saudáveis em AME não precisam de água durante os primeiros seis meses, nem mesmo em dias quentes, ${ }^{(18)}$ denotando que o oferecimento 
desse líquido é comum entre as mães brasileiras, corroborando o presente estudo.

No que diz respeito ao suporte para amamentação, no presente estudo, as mães mais jovens buscaram, no meio social e familiar, informaçóes para guiar as práticas alimentares de seus filhos, o que levou ao oferecimento de outros alimentos antes dos seis meses de vida. Dessa forma, a família, de acordo com a sua história e experiência de vida, transmite para a puérpera seus conhecimentos prévios sobre a alimentação. A influência da avó materna, por exemplo, pode ser um fator negativo para a manutenção do AME até seis meses, visto que em alguns contextos a avó orienta o oferecimento do chá à criança devido a cólicas. ${ }^{(8)}$

Quanto aos motivos da introdução de outro leite no primeiro mês de vida da criança, não houve associação significativa entre mães adolescentes e adultas: os dois grupos, em sua maioria, introduziram o leite artificial por motivos maternos. Sabe-se que as duas primeiras semanas pós-parto são fundamentais para o estabelecimento da amamentação com sucesso. ${ }^{(11)}$ Nessa fase, mãe e bebê estão aprendendo sobre a prática do aleitamento. Assim, esse período é considerado o auge das intercorrências com a amamentação e relatos de que "o leite materno é suficiente, mas não está sustentando a criança", "meu leite é fraco", "o bebê chorava muito", "eu não tive leite", "meu leite secou" são comuns em diversos estudos. ${ }^{\left({ }^{819)}\right.}$ Tais referências ocorrem também com mães adolescentes. ${ }^{(20)}$

Além disso, outro motivo para iniciar alimentação complementar precoce no primeiro mês é o trauma mamilar, o que poderia ser resolvido pela aprendizagem por observação e prática. ${ }^{(21)}$ Dessa forma, é importante que o profissional de saúde auxilie as nutrizes nesse momento, para orientar e sanar dúvidas quanto às técnicas de amamentação e também quanto às recomendaçóes de início oportuno de alimentação complementar. ${ }^{(21)}$ Muitas vezes as justificativas para a introdução precoce de alimentos no primeiro mês de vida do bebê, sob escuta qualificada, podem ser interpretadas como um pedido de ajuda frente às dificuldades desse período. A alegação de leite fraco, por exemplo, pode ser uma justificativa cultural, devido ao desconhecimento das mães quanto às características do leite materno, e ao fato de relacionarem o choro do bebê à fome, o que nem sempre ocorre, pois o choro pode estar relacionado a outras necessidades. ${ }^{(22,23)}$

No que tange à introdução de suco, outros leites, papas e frutas com 90 dias de vida da criança, não houve associação significativa entre os motivos alegados pelas mães adolescentes e adultas. Estudo mostra que aos três meses de idade, em média, ocorre a introduçáo de um novo alimento na dieta da criança, principalmente em países com baixa renda, onde o padrão quase universal é introduzir complementos alimentares. ${ }^{(24)}$ Apesar disso, um estudo canadense evidenciou que, após intervenção efetiva de familiares e profissionais de saúde qualificados durante o aleitamento materno, a mãe adolescente compreendeu a importância do AME e manteve essa prática até os seis meses de vida do bebê. (25) Dessa forma, reafirma-se que os familiares e os profissionais de saúde exercem influência sobre as mães frente aos costumes e experiências prévias de inserção de complemento na dieta das crianças.

Com relação aos motivos alegados pelas participantes para introdução de outro leite com 180 dias, adolescentes e adultas alegaram os mesmos motivos, relacionados ao próprio desejo. Já com relação à introdução de água, chá, suco e papas ou frutas, não houve associação estatística significativa quanto aos motivos alegados. Autores mostram que no sexto mês de vida da criança, o consumo de vários alimentos é aumentado, sendo os principais a água (77,5\%), frutas $(62,7 \%)$, sucos $(57,2 \%)$ e comida de sal $(55,1 \%) .{ }^{(17)}$ Nesse ínterim, a presente pesquisa evidenciou que $15,0 \%$ do total da amostra (mães adolescentes e adultas) conseguiu manter o AME até os seis meses de vida da criança, introduzindo outros alimentos como água, sucos, frutas e papas oportunamente após esse período.

O leite materno supre todas as necessidades nutricionais da criança até os seis meses de vida, não sendo necessário, portanto, introduzir qualquer outro alimento na dieta até esse período. (1,12,24-26) $^{-}$ Entretanto, a partir dos seis meses, é fundamental a introdução oportuna de alimentos complementares, a fim de garantir o adequado padrão de desenvolvimento da criança. ${ }^{(12,27)}$ Assim, a introdução oportu- 
na de alimentos faz com que a criança satisfaça suas necessidades nutricionais, aproxime-se dos hábitos alimentares da família e permite a adequação a uma nova fase do ciclo de vida experimentando novos sabores, cores, texturas e aromas, ${ }^{(27)}$ além de contribuir na prevenção de doenças, no desenvolvimento psicológico, motor e cognitivo. ${ }^{(28)}$ A introdução de alimentos seguros, com nutrientes necessários, além do leite materno, é importante para o desenvolvimento e crescimento infantil. ${ }^{(29,30)}$

Assim, entende-se que o AM não é um ato puramente fisiológico. Trata-se de um fenômeno complexo e, nesse sentido, a decisão da mãe de amamentar ou não envolve fatores sociais, psicológicos e culturais. ${ }^{(31)}$ Diante dessa perspectiva, os familiares e os profissionais de saúde exercem um papel imprescindível no acompanhamento da introdução de alimentos de forma oportuna, já que as mães, por se sentirem inseguras, consideram a orientação destas pessoas. No presente estudo, a maioria das crianças não pode se beneficiar do AME e isso evidencia uma necessidade de se implantar um processo educativo de forma mais participativa, contínua e melhor planejada, desde o período gravídico e que se estenda até o período pós-natal tardio, a fim de melhor capacitar essas mulheres para que sejam mães mais seguras e detentoras dos conhecimentos necessários para a manutenção do aleitamento de seus filhos. O estudo apresentou como limitação a impossibilidade do acompanhamento presencial das participantes nos três momentos da pesquisa; no entanto, o contato via telefone foi de grande importância e capaz de sanar esta limitação.

\section{Conclusão}

Este estudo acompanhou mães adolescentes e adultas com 30, 90 e 180 dias pós-parto, permitindo dar maior visibilidade ao cuidado dispensado por elas quando alimentam seus bebês. As adolescentes e adultas apresentaram motivos diferentes para a introdução de chá aos 30 dias pós-parto, para a introdução de água e chá aos 90 dias e apresentaram o mesmo motivo para a introdução de outro leite aos 180 dias pós-parto. As adolescentes recorrem à orientação de outras pessoas, razão pela qual enfermeiros, médicos e outros profissionais de saúde devem ser preparados em sua formação para lidar com essas especificidades das adolescentes no que tange à maternidade nessa fase da vida. Sugere-se que novas pesquisas sejam realizadas com o acompanhamento da amamentação e alimentação complementar após o sexto mês de vida da criança, entre mães adolescentes e adultas, em especial com a utilização de abordagem qualitativa, com o intuito de responder as lacunas ainda existentes sobre essa temática, contribuindo, assim, para o aumento das taxas do AM.

\section{Colaborações}

Murari CPC, Arciprete APR, Gomes-Sponholz F e Monteiro JCS declaram que contribuíram com a concepção do projeto, análise e interpretação dos dados, redação do artigo, revisão crítica relevante do conteúdo intelectual e aprovaçáo da versáo final a ser publicada.

\section{Referências}

1. Saffari $M$, Pakpour $A H$, Chen $H$. Factors influencing exclusive breastfeeding among Iranian mothers: A longitudinal population-based study. Health Promot Perspect. 2016;7(1):34-41.

2. World Health Organization (WHO). Global Network of Institutions for Scientific Advice on Nutrition. Report of the first Meeting 11-12. Genève: WHO; 2010.

3. Barbosa MB, Palma D, Domene SM, Taddei JA, Lopez FA. Fatores de risco associados ao desmame precoce e ao período de desmame em lactentes matriculados em creches. Rev Paul Pediatr. 2009;27(3):27281.

4. Souto DC, Jager ME, Dias AC. Aleitamento materno e a ocorrência do desmame precoce em puérperas adolescentes. Rev Aten Saúde. 2014;12(41):73-9.

5. Queluz MC, Pereira MJ, dos Santos CB, Leite AM, Ricco RG. Prevalência e determinantes do aleitamento materno exclusivo no município de Serrana, São Paulo, Brasil. Rev Esc Enferm USP. 2012;46(3):537-43.

6. Hector D, King L. Interventions to encourage and support breastfeeding. N S W Public Health Bull. 2005;16(3-4):56-61.

7. Akré J. Alimentação infantil: bases fisiológicas. Genebra: OMS; 1994.

8. Oliveira CS, locca FA, Carrijo ML, Garcia RA. Breastfeeding and complications that contribute to early weaning. Rev Gaúcha Enferm. 2015;36(Spe):16-23.

9. World Health Organization (WHO). Health for the world's adolescents: a second chance in the second decade. Genève: WHO; 2014. 
10. Clapis CV, Fabbro MR, Beretta MI. The practice of breastfeeding of teen mothers in the first six months of life of the child. Cienc Cuid Saude. 2013;12(4):704-10.

11. Brasil. Ministério da Saúde. Secretaria de Atenção à Saúde. Departamento de Atenção Básica. Saúde da criança: aleitamento materno e alimentação complementar. Brasília (DF): Ministério da Saúde; 2015.

12. United Nations Children's Fund (UNICEF). Improving child nutrition - The achievable imperative for global progress. New York: UNICEF; 2013.

13. Core Team. R: A language and environment for statistical computing. Viena: R Foundation for Statistical Computing; 2017. A

14. Alvisi P, Brusa S, Alboresi S, Amarri S, Bottau P, Cavagni G, et al. Recommendations on complementary feeding for healthy, full-term infants. Ital J Pediatr. 2015;41(1):36.

15. Nasreddine L, Zeidan MN, Naja F, Hwalla N. Complementary feeding in the MENA region: practices and challenges. Nutr Metab Cardiovasc Dis. 2012;22(10):793-8.

16. Schincaglia RM, Oliveira AC, Sousa LM, Martins KA. Práticas alimentares e fatores associados à introdução precoce da alimentação complementar entre crianças menores de seis meses na região noroeste de Goiânia. Epidemiol Serv Saude. 2015;24(3):465-74.

17. Campos AM, Chaoul CO, Carmona EV, Higa R, Vale IN. Prática de aleitamento materno exclusivo informado pela mãe e oferta de líquidos aos seus filhos. Rev Lat Am Enfermagem. 2015;23(2):283-90.

18. World Health Organization (WHO); United Nations Children's Fund (UNICEF). Baby-friendly hospital initiative: revised, updated and expanded for integrated care. Section1, Background and implementation. Genève: WHO; 2009.

19. Brown CR, Dodds L, Legge A, Bryanton J, Semenic S. Factors influencing the reasons why mothers stop breastfeeding. Can J Public Health. 2014;105(3):e179-85.

20. Souza SA, Araújo RT, Teixeira JR, Mota TN. Aleitamento materno: fatores que influenciam o desmame precoce entre mães adolescentes. Rev Enferm UFPE. 2016;10(10):3806-13.
21. de Almeida JM, Luz SA, Ued FV. Apoio ao aleitamento materno pelos profissionais de saúde: revisão integrativa da literatura. Rev Paul Pediatr. 2015;33(3):356-63.

22. Moreira ASH, Murara AZ. Aleitamento materno, desmame precoce e hipogalactia: o papel do nutricionista. Rev Eletrôn Faculdade Evangélica do Paraná. 2012;2 (2):51-61.

23. Rocci E, Fernandes RA. Dificuldades no aleitamento materno e influencia no desmame precoce. Rev Bras Enferm. 2014;67(1):227.

24. Greiner T. Exclusive breastfeeding: measurement and indicators. Int Breastfeed J. 2014;9(18):18.

25. Meglio GD, McDermott MP, Klein JD. A randomized controlled trial of telephone peer support's influence on breastfeeding duration in adolescent mothers. Breastfeed Med. 2010;5(1):41-7.

26. Qasem W, Fenton T, Friel J. Age of introduction of first complementary feeding for infants: a systematic review. BMC Pediatr. 2015;15(107):107.

27. Brasil. Ministério da Saúde. Secretaria de Atenção à Saúde. Saúde da criança: aleitamento materno e alimentação complementar. 2a ed. Brasília (DF): Ministério da Saúde; 2015.

28. Koletzko B, Bhatia J, Bhutta ZA, Cooper P, Makrides M, Uauy R, et al. Pediatric nutrition in practice. 2nd ed. Munich: Karger; 2015. Vol. 113.

29. Bhutta ZA, Das JK, Rizvi A, Gaffey MF, Walker N, Horton S, et al.; Lancet Nutrition Interventions Review Group, the Maternal and Child Nutrition Study Group. Evidence-based interventions for improvement of maternal and child nutrition: what can be done and at what cost? Lancet. 2013;382(9890):452-77.

30. Victor R, Baines SK, Agho KE, Dibley MJ. Factors associated with inappropriate complementary feeding practices among children aged 6-23 months in Tanzania. Matern Child Nutr. 2014;10(4):54561.

31. Azevedo AR, Alves VH, Souza RM, Rodrigues DP, Branco MB, Cruz AF. Clinical management of breastfeeding: knowledge of nurses. Esc Anna Nery. 2015;19(3):439-45. 